\title{
CMEARTICLE
}

\section{Clinics in diagnostic imaging (195)}

Felicia $\underline{T e O}^{1}$, MBBS, Mohammad Taufik bin Mohamed Shah ${ }^{2}$, MBBS, FRCR, Bak Siew Steven Wong ${ }^{2}$, MBChB, MMed
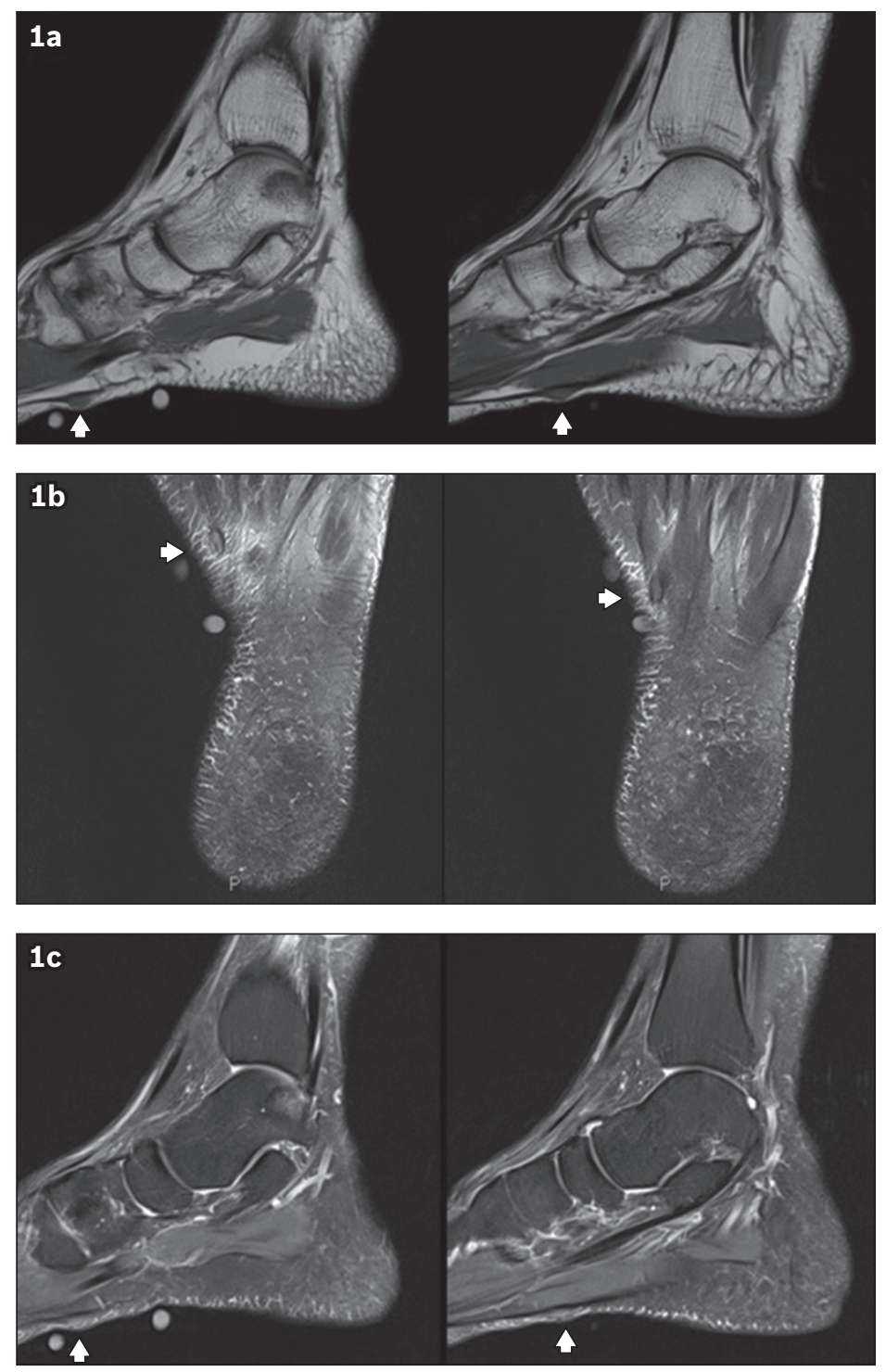

Fig. 1 (a) Sagittal T1-W, (b) axial T2-W fat saturation and (c) sagittal proton density-weighted fat saturation MR images of the left foot.

\section{CASE PRESENTATION}

A 46-year-old man presented with a six-month history of lumps in the sole of his left foot. They were associated with pain upon bearing weight. He had a history of Dupuytren's contracture involving his right hand, which had been surgically treated
11 years ago. Physical examination revealed two palpable plantar lumps, one firm and one tender, in his left foot with no overlying skin changes. Initial plain radiographs showed no abnormality. He subsequently underwent magnetic resonance (MR) imaging (Fig. 1). What do the images show? 


\section{IMAGE INTERPRETATION}

Non-contrast-enhanced MR imaging of the left foot revealed two sub-centimetre nodules (arrowheads, Fig. 1a-c) along the medial band of the plantar fascia (i.e. aponeurosis). The nodules are isointense relative to muscle on the $\mathrm{T} 1$-weighted sequences (Fig. 1a) and isointense to slightly hyperintense on T2-weighted sequences (Fig. 1b). They are hyperintense on the proton densityweighted and fat-saturation sequences (Fig. 1c).

\section{DIAGNOSIS}

Plantar fibromatosis.

\section{CLINICAL COURSE}

As the nodules caused symptoms, the patient opted for surgical management. A wide excision was performed with good postoperative recovery. No nodule recurrence was reported one year after surgical excision. Histological evaluation of the excised nodules showed dense fibrocollagenous fascial tissues with nodular and infiltrative proliferation of bland spindle cells in large interlacing fascicles, consistent with plantar fibromas.

\section{DISCUSSION}

The plantar fascia, or aponeurosis, is a strong layer of fibrous tissue in the sole of the foot. It stabilises the medial longitudinal arch of the foot and consists of three bundles (or bands): medial, central and lateral. Posteriorly, it is attached to the medial calcaneal tubercle. Anteriorly, it has a complex insertion at the level of the metatarsal heads. ${ }^{(1)}$

Plantar fibromatosis, or Ledderhose disease, is a hyperproliferative disorder of mature fibroblasts that causes nodular thickening of the plantar aponeurosis. ${ }^{(2)}$ Although benign, it may invade the overlying skin or deeper structures such as the adjacent plantar muscles and tendon sheaths. ${ }^{(3)}$ There is no clear correlation between Ledderhose disease and malignant transformation. However, our search of the English medical literature revealed one reported case of squamous cell carcinoma arising from long-standing plantar fibromas. ${ }^{(4)}$

The exact aetiology of plantar fibromatosis is unknown; however, it is related to palmar fibromatosis (i.e. Dupuytren's contracture $)^{(2,5)}$ and fibromatosis-like conditions such as Peyronie's disease. ${ }^{(2,5)}$ Several conditions proposed as risk factors to developing plantar fibromatosis include: underlying genetic predisposition; prior history of trauma; and underlying medical conditions such as diabetes mellitus and epilepsy..$^{(2,3,5,6)}$ Disease onset typically occurs between the ages of 30 and 50 years. ${ }^{(3)}$ However, children and pre-adolescents may be affected. ${ }^{(5)}$ Higher incidences of the condition have been reported in Caucasians ${ }^{(4)}$ and in men. ${ }^{(2,3)}$

Patients usually present with a palpable slow-growing nodule at the medial aspect of the sole. Some may complain of multiple nodules. There is bilateral involvement in about $25 \%-50 \%$ of patients. (2) Patients with larger nodules may also report pain after long periods of walking or standing. ${ }^{(2,3)}$ Pain occurs in two ways: (a) from nodule compression upon weight bearing, which is further aggravated by the presence of surrounding soft tissue

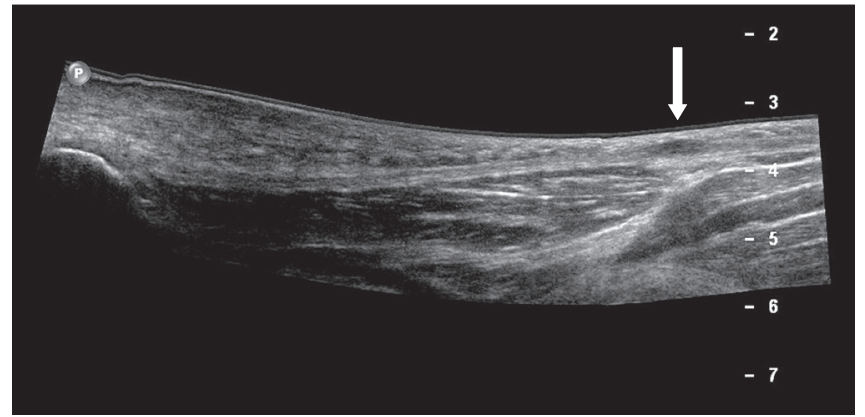

Fig. 2 A 43-year-old man presented with left foot pain. Longitudinal US image of the sole of the medial left foot shows the uniform fibrillar structure of the normal plantar aponeurosis. There is a hypoechoic fusiform nodule (arrow) that is inseparable from the plantar aponeurosis, in keeping with a plantar fibroma.

inflammation, and (b) from local invasion of the adjacent muscle or neurovascular structures. ${ }^{(3)}$ On physical examination, nodules are typically firm and fixed, located at the medial plantar midfoot, and may be adherent to the skin. ${ }^{(3)}$ Contractures are an uncommon feature in plantar fibromatosis, as opposed to palmar fibromatosis. $^{(3)}$

Although the diagnosis of plantar fibromatosis is generally established clinically, imaging helps to improve diagnostic confidence. The value of imaging to the diagnostic work-up is in its ability to identify and distinguish plantar fibromas from other conditions with similar presentations. It is able to uncover occult conditions that might change the clinical course and management of the patient. In imaging evaluation, an initial radiograph is usually not required ${ }^{(2)}$ as it is frequently normal. ${ }^{(3)}$ However, radiographs may be helpful when there is clinical suspicion of bony abnormalities such as fractures, exostoses or other bone-forming neoplasms. The utility of radiography in plantar fibromatosis is in detecting contour-deforming soft tissue masses and, in larger nodules, helping to identify the presence of adjacent bony erosions. As such, the modalities of ultrasonography (US) and MR imaging are favoured over plain radiography for diagnosis as they provide superior soft tissue contrast.

Given the superficial nature of the nodules, US is a useful and inexpensive modality for evaluation and characterisation. US provides real-time dynamic imaging with good spatial resolution. It can differentiate solid from cystic structures within soft tissue. More importantly, US provides direct patient interaction; the clinician or radiologist can immediately correlate sonographic finding with the patients' symptom. Sonographically, the normal plantar aponeurosis appears as a uniform band of echogenic fibres within a hypoechoic-to-anechoic background, giving rise to its fibrillar pattern. In contrast, fibromas appear as fusiform hypoechoic nodules on the plantar surface or within the substance of the plantar aponeurosis. ${ }^{(7)}$ For accurate diagnosis, it is important to visualise nodule continuity with the plantar aponeurosis ${ }^{(8)}$ (Fig. 2). Larger lesions (>10 mm) have a rounder appearance and are more likely to demonstrate inhomogeneous echoes ${ }^{(7)}$ (Fig. 3a). Nodules may also show increased vascularity on colour Doppler evaluation $^{(3,8)}$ (Fig. 3b). Small intranodular cystic components causing posterior acoustic enhancement may also be present; 

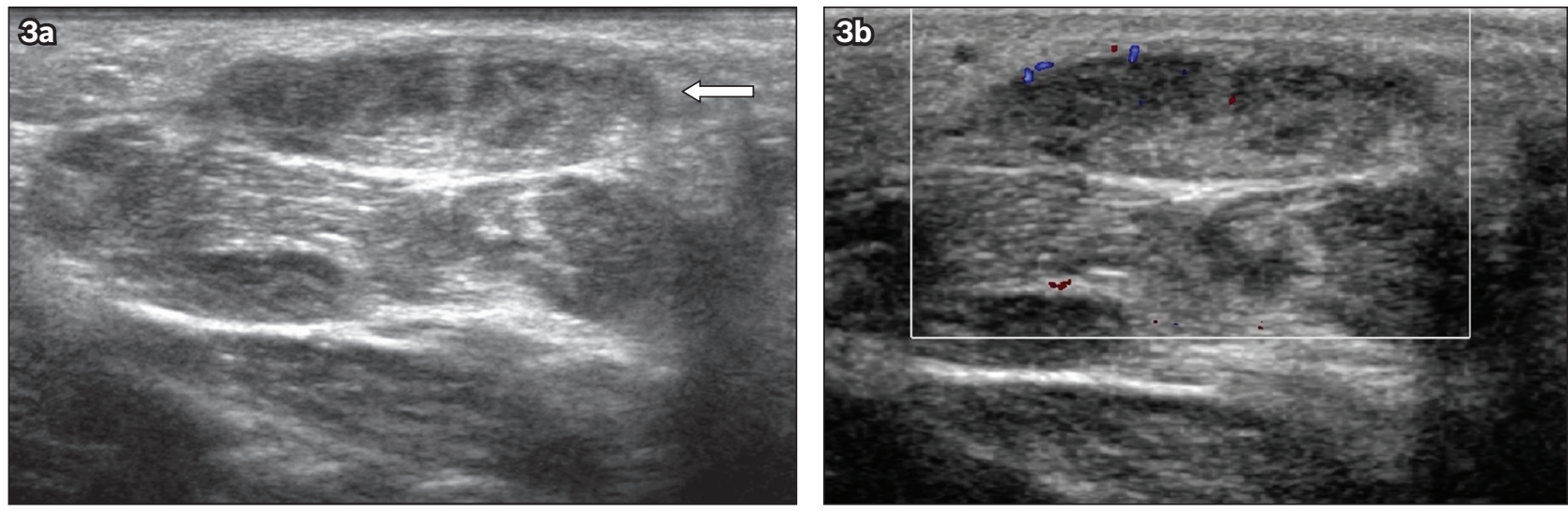

Fig. 3 A 53-year-old man presented with a plantar lump. (a) Transverse greyscale and (b) colour Doppler US images of the sole of his right foot show a heterogeneous nodule arising from the medial plantar aponeurosis (arrow), which shows mild increased internal vascularity on colour Doppler imaging. The nodule was subsequently excised, and the diagnosis of plantar fibroma was confirmed histologically.

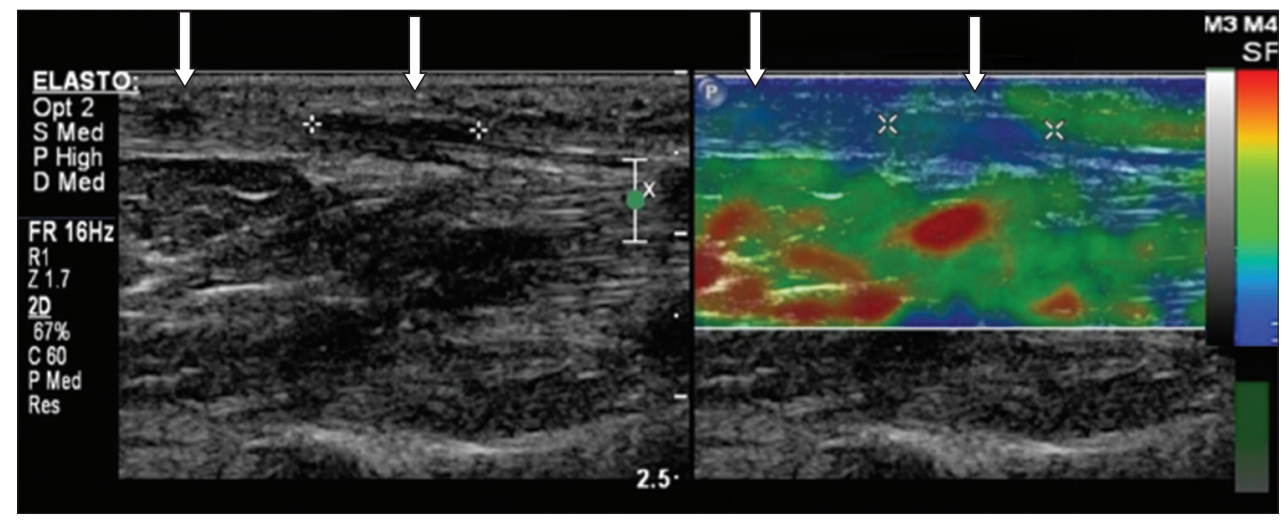

Fig. 4 A 57-year-old man presented with pain in the plantar aspect of his left midfoot. Longitudinal US image with dual modalities (left: greyscale, right: strain sonoelastography) shows two plantar fibromas (arrows) with reduced relative elasticities (blue) compared to the adjacent normal plantar fascia (green).

Haun et al in 2011 reported a case of plantar fibroma that showed myxoid stromal degeneration and cysts histologically. ${ }^{(9)}$

Sonoelastography is useful as a supplementary technique in musculoskeletal imaging. It provides qualitative information about the relative elasticities of soft tissues by measuring how they respond to applied stresses, using either shear-wave propagation or strain application (i.e. compression). The former technique calculates the velocity of shear-wave propagation in tissues, then converts them into colour-coded maps, called elastograms, that assign relative local tissue elasticity (or 'stiffness'). The latter technique compares differences in time for the ultrasonography waves to return to the transducer after manual compressive pressures are applied. Conventionally, the colour red indicates the presence of more elastic (or 'soft') tissues, while blue indicates less elastic (or 'stiff') ones; those of intermediate relative stiffness are assigned a colour within the spectrum of green or yellow. Recently, several studies have demonstrated the usefulness of sonoelastography in differentiating between benign and malignant soft tissue masses and in characterising the type of benign masses. ${ }^{(10)}$ Its use in plantar fasciitis showed that the fascia in affected individuals was more elastic (or 'softer') compared to healthy subjects. While the utility of sonoelastography in plantar fibromas has yet to be established, we have anecdotally demonstrated that the nodules are less elastic (or 'stiffer') relative to normal fascia, likely attributable to their fibrous composition (Fig. 4). Taljanovic et al demonstrated in 2017 that fibromas, albeit the palmar variant, show increased relative stiffness when compared to the normal tendon sheath from which they arise. ${ }^{(11)}$ We feel that sonoelastography is a promising technique to help discern between normal and abnormal fascia, although local studies are needed to establish its usefulness.

On MR imaging, the normal plantar aponeurosis is homogeneously hypointense on both T1-weighted and T2-weighted sequences. Plantar fibromas may be heterogeneous, hypointense or isointense (relative to muscle) on both T1-weighted and T2-weighted sequences, depending on the extent of fibrous tissue composition ${ }^{(1,12,13)}$ (Fig. 5). They are typically hyperintense on proton density-weighted fat saturation sequences $^{(3)}$ (Fig. 1C). Nodules that are more aggressive show invasion of deeper structures and are frequently hyperintense on T2-weighted sequences; it is postulated that this is due to higher cellular matrix components. ${ }^{(13)}$ The nodules show variable enhancement after administration of intravenous gadoliniumbased contrast media ${ }^{(12,13)}$ (Fig. 6). Contrast may improve the sensitivity of detecting smaller nodules, especially where small hypointense nodules may be difficult to differentiate from low-signal aponeurosis on non-contrast-enhanced images. ${ }^{(1,7)}$ Such nodules may be clinically missed, even with sonographic evaluation. Finally, MR imaging is superior in evaluating the 



Fig. 5 A 56-year-old woman presented with a palpable and painful lump in the plantar aspect of her right foot. (a) Sagittal T1-W and (b) T2-W fat-saturation MR images of the right foot show a fusiform nodule with intermediate T1-W and T2-W signal centred at the medial plantar fascia (arrows), consistent with plantar fibroma.
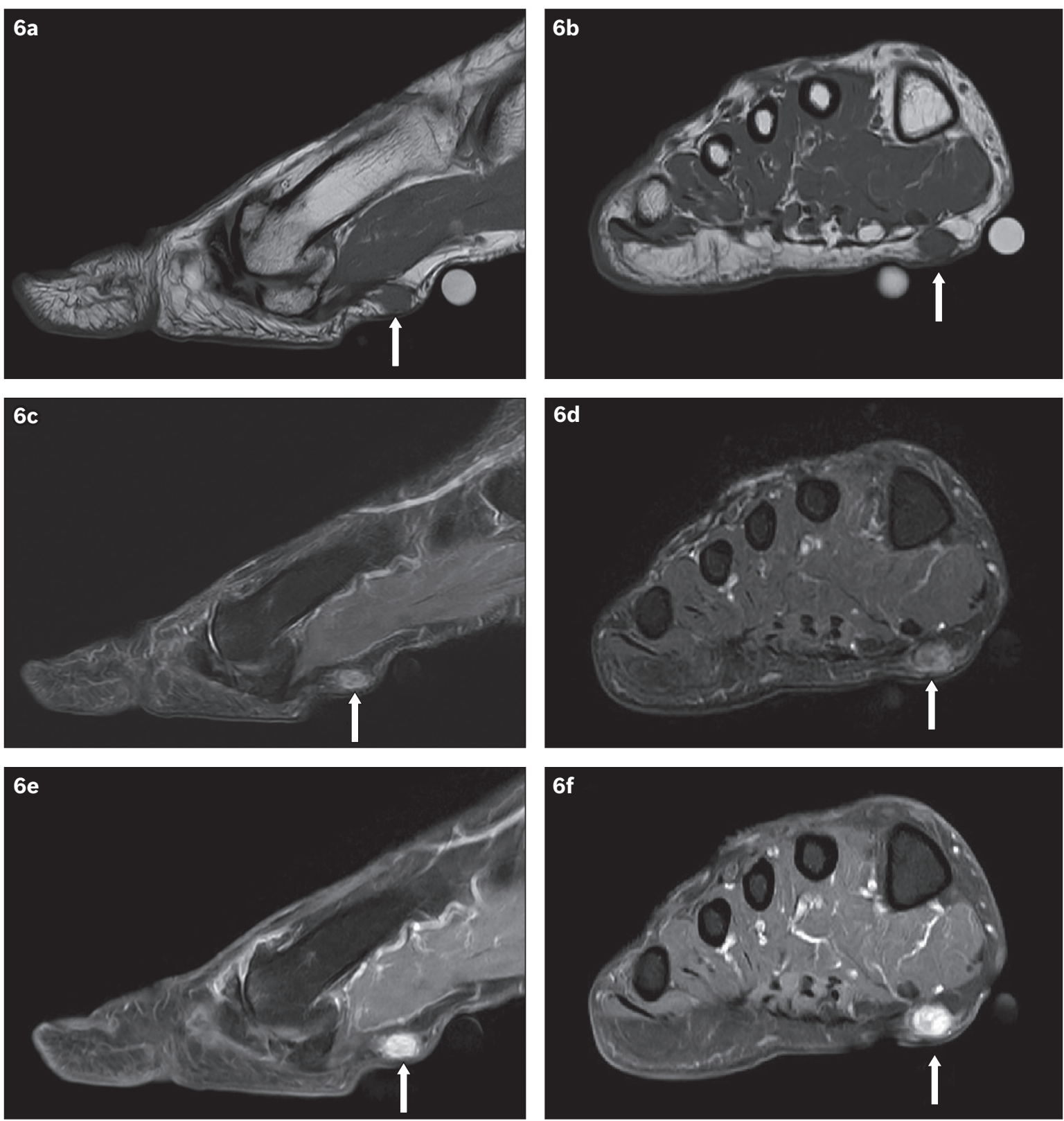

Fig. 6 A 58-year-old man presented with a six-month history of a right plantar fibroma (arrows). (a) Sagittal and (b) coronal T1-W MR sequences of the left foot show an isointense nodule at the medial aspect of the plantar fascia. This nodule is heterogeneously hyperintense on (c) sagittal and (d) coronal T2-W fat saturation sequences. (e) Sagittal and (f) coronal T1-W fat saturation images show avid nodule enhancement after administration of intravenous gadolinium-based contrast media. 
extent of deep tissue invasion and is the modality of choice when this is clinically suspected. ${ }^{(13)}$

In the evaluation of a plantar mass and/or painful sole, there are other benign and malignant conditions that should be considered in the differential diagnoses. Benign conditions include ganglion cysts, neurogenic tumours (e.g. neurofibromas), intermetatarsal (or Morton) neuromas and mesenchymal tumours (e.g. leiomyomas). The possibility of malignant soft tissue lesions, such as rhabdomyosarcomas and liposarcomas, should be contemplated when encountering patients with more aggressive clinical presentations. Imaging, in particular MR imaging, can help confirm and differentiate plantar fibromas from the above differentials.

Other common benign conditions related to the aponeurosis include plantar fasciitis, chronic aponeurotic rupture ${ }^{(8)}$ and foreign body reaction. ${ }^{(12)}$ In plantar fasciitis, thickening of the aponeurosis usually occurs at its calcaneal attachment and is related to a bony plantar calcaneal spur. This contrasts with plantar fibromas, which usually arise in the plantar midfoot. A prior history of trauma may be elicited in chronic aponeurotic ruptures. A detailed clinical examination can diagnose the tear and detect the presence of surrounding midfoot oedema. Also, a chronically ruptured plantar aponeurosis may be focally thickened $^{(8)}$ and easily detected on US. Similarly, when a plantar foreign body is suspected, US or MR imaging can help confirm the diagnosis. Foreign bodies are typically hyperechoic on US. ${ }^{(12)}$ On MR imaging, they are commonly hypointense on T1-weighted with surrounding hyperintense T2-weighted signal due to granulation tissue. ${ }^{(12)}$

Treatment for plantar fibromatosis depends on symptom severity and extent of the nodules. When pain is mild and the nodules are small and not rapidly enlarging, management is primarily conservative. ${ }^{(2)}$ At this stage, orthotic inserts and antiinflammatory medications such as nonsteroidal anti-inflammatory drugs may be prescribed to relieve pain from weight bearing. The patient may be given local steroid injections percutaneously or radiotherapy when the pain does not improve. These treatments work by decreasing fibroblastic proliferation, hence reducing the size and therefore pain that results from the nodules. ${ }^{(2)}$ Surgery is reserved for patients with worsening symptoms and progressively enlarging nodules, usually after a period of conservative treatment. ${ }^{(2,3)}$ Various techniques have been described for surgical removal. These include local excision, wide resection, and partial or total plantar fasciectomy. ${ }^{(14)}$ However, plantar fibromas are notorious for recurring post excision due to their infiltrative growth pattern. ${ }^{(3,8)}$ Their recurrence rates are also inversely proportional to the extent of excision, with the highest recurrence rates reported after local excision and lowest after total plantar fasciectomy. ${ }^{(5,14,15)}$ Higher recurrence is also seen in patients with a positive family history and those with multiple nodules and bilaterality (i.e. affecting both feet) ${ }^{(15)}$ - these nodules also tend to be more aggressive. ${ }^{(13,15)}$ In addition to surgical therapy, adjuvant radiotherapy may help lower recurrence but may be associated with higher morbidity. ${ }^{(14,15)}$ Other investigational treatment approaches that have been reported in the literature include extracorporeal shock wave therapy and anti-oestrogen therapy. ${ }^{(2)}$

In conclusion, while imaging helps to increase the physician's confidence in diagnosing plantar fascia, it is also useful in detecting mimicking conditions that present similarly, or as a problem-solving tool in more challenging clinical encounters. More importantly, the reasons for imaging must be clear to avoid unnecessary costs related to increasingly expensive evaluations.

ABSTRACT A 46-year-old man presented with a six-month history of lumps in the sole of his left foot. Physical examination revealed two nodules, one tender and one firm, at the plantar left foot with no overlying skin changes. Although the initial radiographs were normal, magnetic resonance imaging of the left foot demonstrated two nodules along the medial band of the plantar fascia, characteristic of plantar fibromas. The patient opted for surgical excision. There was no further recurrence of symptoms after surgery. We describe the clinical and radiological features of plantar fibromatosis and briefly discuss other causes of lumps and pain in the sole of the foot.

Keywords: Ledderhose disease, magnetic resonance imaging, plantar fibromatosis, plantar pain, ultrasonography

\section{REFERENCES}

1. McNally EG, Shetty S. Plantar fascia: imaging diagnosis and guided treatment. Semin Musculoskelet Radiol 2010; 14:334-43.

2. Veith NT, Tschernig T, Histing T, Madry H. Plantar fibromatosis--topical review. Foot Ankle Int 2013; 34:1742-6.

3. Murphey MD, Ruble CM, Tyszko SM, et al. From the archives of the AFIP: musculoskeletal fibromatoses: radiologic-pathologic correlation. Radiographics 2009; 29:2143-73.

4. Motolese A, Mola F, Cherubino M, et al. Squamous cell carcinoma and ledderhose disease: a case report. Int J Low Extrem Wounds 2013; 12:297-300.

5. Fetsch JF, Laskin WB, Miettinen M. Palmar-plantar fibromatosis in children and preadolescents: a clinicopathologic study of 56 cases with newly recognized demographics and extended follow-up information. Am J Surg Pathol 2005; 29:1095-105.

6. Elhadd TA, Ghosh S, Malik MI, Collier A. Plantar fibromatosis and Dupuytren's disease: an association to remember in patients with diabetes. Diabet Med 2007; 24:1305.

7. Bedi DG, Davidson DM. Plantar fibromatosis: most common sonographic appearance and variations. J Clin Ultrasound 2001; 29:499-505.

8. Griffith JF, Wong TY, Wong SM, Wong MW, Metreweli C. Sonography of plantar fibromatosis. AJR Am J Roentgenol 2002; 179:1167-72.

9. Haun DW, Cho JC, Kettner NW. Symptomatic plantar fibroma with a unique sonographic appearance. J Clin Ultrasound 2012; 40:112-4.

10. Klauser AS, Miyamoto $\mathrm{H}$, Bellmann-Weiler R, et al. Sonoelastography: musculoskeletal applications. Radiology 2014; 272:622-33.

11. Taljanovic MS, Gimber LH, Becker GW, et al. Shear-wave elastography: basic physics and musculoskeletal applications. Radiographics 2017; 37:855-70.

12. Jeswani T, Morlese J, McNally EG. Getting to the heel of the problem: plantar fascia lesions. Clin Radiol 2009; 64:931-9.

13. Morrison WB, Schweitzer ME, Wapner KL, Lackman RD. Plantar fibromatosis: a benign aggressive neoplasm with a characteristic appearance on MR images. Radiology 1994; 193:841-5

14. van der Veer WM, Hamburg SM, de Gast A, Niessen FB. Recurrence of plantar fibromatosis after plantar fasciectomy: single-center long-term results. Plast Reconstr Surg 2008; 122:486-91.

15. de Bree E, Zoetmulder FA, Keus RB, Peterse HL, van Coevorden F. Incidence and treatment of recurrent plantar fibromatosis by surgery and postoperative radiotherapy. Am J Surg 2004; 187:33-8. 


\section{SINGAPORE MEDICAL COUNCIL CATEGORY 3B CME PROGRAMME} (Code SMJ 201905B)

Question 1. Regarding plantar fibromatosis:

(a) It is more common in male patients.

(b) It is not seen in children.

(c) It is related to Dupuytren's contracture and Peyronie's disease.

(d) Diabetes mellitus is a risk factor.

Question 2. Typical presenting features of plantar fibromatosis include:

(a) Plantar contractures.

(b) Bilateral plantar lumps.

(c) Solitary plantar nodule.

(d) Chronic discharging sinus at the plantar aspect of the foot.

Question 3. The following is the best imaging modality for diagnosis of plantar fibromatosis:
(a) Plain radiography.
(b) Computed tomography.
(c) Magnetic resonance imaging.
(d) Positron-emission tomography-computed tomography.

Question 4. The following is a diagnostic finding for plantar fibromas on ultrasonography:

(a) Fusiform hypoechoic nodules within the plantar aponeurosis at the mid-foot region.

(b) Rounded hypoechoic nodule in the intermetatarsal space.

(c) A uniform band of echogenic fibres within a hypoechoic background matrix.

(d) Plantar fibromas may show increased vascularity on colour Doppler imaging.



Question 5. Magnetic resonance imaging has the following advantage(s) over ultrasonography in the evaluation of plantar fibromas:

(a) It allows the operator to immediately correlate radiological findings with clinical symptoms.

(b) It allows visualisation of the fibrillar architecture of the normal plantar aponeurosis.

(c) Compared to ultrasonography, smaller lesions are less likely to be missed.

(d) It is excellent at demonstrating the extent of invasion into deeper structures.

\section{Doctor's particulars:}

Name in full:

MCR no.:

Specialty:

Email:

\footnotetext{
SUBMISSION INSTRUCTIONS:

Visit the SMJ website: http://www.smj.org.sg/current-issue and select the appropriate quiz. You will be redirected to the SMA login page.

For SMA member: (1) Log in with your username and password (if you do not know your password, please click on 'Forgot your password?'). (2) Select your answers for each quiz and click 'Submit'.

For non-SMA member: (1) Create an SMJ CME account, or log in with your SMJ CME username and password (for returning users). (2) Make payment of SGD 21.40 (inclusive of $7 \%$ GST) via PayPal to access this month's quizzes. (3) Select your answers for each quiz and click 'Submit'.

RESULTS:

(1) Answers will be published online in the SMJ July 2019 issue. (2) The MCR numbers of successful candidates will be posted online at the SMJ website by 10 July 2019. (3) Passing mark is $60 \%$. No mark will be deducted for incorrect answers. (4) The SMJ editorial office will submit the list of successful candidates to the Singapore Medical Council. (5) One CME point is awarded for successful candidates. (6) SMC credits CME points according to the month of publication of the CME article (i.e. points awarded for a quiz published in the December 2017 issue will be credited for the month of December 2017, even if the deadline is in January 2018).

Deadline for submission (May 2019 SMJ 3B CME programme): 12 noon, 3 July 2019.
} 